\title{
ESTUDIO ANATOMICO DE LA MADERA DE PSIDIUM SARTORIANUM (MYRTACEAE) Y CORDIA GERASCANTHUS (BORAGINACEAE) ${ }^{1}$
}

\author{
Silvia Rebollar \\ Alejandra Quintanar \\ Carmen de la Paz Perez \\ Departamento de Biología \\ División de Ciencias Biológicas y de la Salud \\ Universidad Autónoma Metropolitana-Iztapalapa \\ Apdo. Postal 55-535 \\ 09340 México, D.F.
}

\section{RESUMEN}

Se describen las características anatómicas macroscópicas y microscópicas de Psidium sartorianum (Berg.) Ndzu. y Cordia gerascanthus L., procedentes de una selva mediana subperennifolia en Puerto Morelos, Municipio de Benito Juárez, Quintana Roo. Se dan los valores cuantitativos de los elementos mensurables. Se incluye la descripción botánica de las especies, las características de los árboles estudiados así como los usos locales y los referidos en la literatura. Se discuten los resultados obtenidos.

\section{ABSTRACT}

Macroscopic and microscopic anatomical features of the wood of Psidium sartorianum (Berg.) Ndzu. and Cordia gerascanthus L. are described. The samples were collected in tropical rain forests of Puerto Morelos, Municipio of Benito Juárez, Quintana Roo. Values of measurable elements, botanical descriptions of each species, and characteristics of the trees are reported, as also local uses and those mentioned in the literature. The results of these studies are discussed.

\section{INTRODUCCION}

La selva alta y mediana subperennifolia comprende una gran extensión del estado de Quintana Roo (Miranda, 1978), con una diversidad de especies interesantes para su estudio. Tal es el caso de las maderables que en su mayoría son desconocidas en sus características estructurales y tecnológicas (Rebollar et al., 1987), algunas de las cuales por sus dimensiones y abundancia pueden ser consideradas como potencialmente comerciales (Escalante, 1986). Para este estudio se seleccionaron dos de esas especies que son muy usadas localmente en el Municipio de Benito Juárez: Psidium sartorianum (Berg.) Ndzu. y Cordia gerascanthus L., contribuyendo así al conocimiento de su estructura anatómica.

${ }^{1}$ Este trabajo tuvo el apoyo económico de la Secretaría de Educación Pública, convenio 086-01-0261. 
Los estudios de la madera realizados en México para estos dos géneros contemplan aspectos descriptivos de las características anatómicas, usos, así como algunos datos tecnológicos.

Para Psidium se conoce la contribución correspondiente a $P$. guajava de Veracruz (Ortega et al., 1988) y la que se ocupa de $P$. sartorianum de Jalisco (Barajas y León, 1989).

Para el género Cordia se encuentran los siguientes trabajos: el referente a $C$. dodecandra de Campeche (Guridi, 1968); los que atañen a C. alliodora de Chiapas (Ortega, 1958; Andrade, 1976 y Ortega, 1984) y de Jalisco (Barajas y Echenique, 1976), y el que se ocupa de C. sonorae de Jalisco (Barajas y Echenique, 1976). El estudio más amplio de la familia Boraginaceae (Barajas, 1981) incluye las siguientes especies: C. alliodora, C. dentata, $C$. elaeagnoides, $C$. hintonii y $C$. sonorae, procedentes de Jalisco y finalmente existen dos trabajos para C. elaeagnoides: uno de Michoacán (Guridi, 1980) y otro de Jalisco (León y Barajas, 1987).

\section{MATERIAL Y METODOS}

Los árboles estudiados fueron recolectados por las dos primeras autoras de acuerdo con las instrucciones de Ramos y Díaz (1981), en los terrenos del Jardín Botánico "Dr. Alfredo Barrera Marín" del Centro de Investigaciones de Quintana Roo en Puerto Morelos. Las muestras botánicas fueron identificadas y registradas en el Herbario Metropolitano de la Universidad Autónoma Metropolitana Iztapalapa.

Se seleccionó un árbol maduro y sano para cada especie; de cada uno se obtuvieron tablillas de xiloteca de $15 \times 7 \times 1 \mathrm{~cm}$, con las que se describieron las características macroscópicas y dos rodajas de $2 \mathrm{~cm}$ de grosor cada una, de donde se tomaron al azar cubos de $2 \times 2 \mathrm{~cm}$, para la obtención de cortes típicos de la madera y material disociado, con los que se hizo el estudio de los rasgos microscópicos.

Los cubos se sometieron a un proceso de ablandamiento de acuerdo con Kukachka (1977); los cortes se hicieron en un microtomo de deslizamiento, se tiñeron con verde yodo, se deshidrataron y montaron con resina sintética. El material disociado se obtuvo por el método de Jeffrey (Johansen, 1940), se tiñó con pardo de Bismarck y se montó con gelatina glicerinada.

La denominación de las características macroscópicas se hizo siguiendo las tablas de Tortorelli (1956) y para el color se usaron las cartas de Munsell (1954). La nomenclatura utilizada en las descripciones microscópicas fue la de IAWA Committee (1989); para los rayos tambien se usó la clasificación de Kribs (1968). A los caracteres mensurables se les hizo un análisis estadístico con un error de muestreo de $5 \%$. Para cada carácter se da el valor de la media y, entre paréntesis, el mínimo y el máximo; su denominación se hizo con base en la media considerando la clasificación de Chattaway (1932) y IAWA Committee (1937, 1939). Para cada especie se presenta además de las características macroscópicas y microscópicas de la madera, la descripción botánica y las características del árbol estudiado así como información sobre los usos locales proporcionada por los campesinos de la localidad y los citados en la literatura. 
Rebollar et al.: Anatomía de la Madera de Psidium sartorianum y Cordia gerascanthus

\section{RESULTADOS}

1. Psidium sartorianum (Berg.) Ndzu.

Myrtaceae.

Nombres comunes

Guayabillo (Guerrero, Oaxaca, Chiapas, Yucatán); arrayán (Sinaloa, Veracruz, Oaxaca, Durango); pichiché (maya, Yucatán); rayana (Oaxaca); choquey (guarigio, Chihuahua) (Pennington y Sarukhán, 1968).

Distribución en México

Se encuentra en la vertiente del Golfo, desde Veracruz hasta el norte de Chiapas, sur de Tabasco y la península de Yucatán, así como en la vertiente del Pacífico, desde Sonora hasta Chiapas, incluyendo la Cuenca del Rio Balsas (Sánchez, 1990).

Descripción botánica de la especie (Fig. 1)

Arbol de 10 a 15 m de altura; hojas elípticas a ovado lanceoladas, aromáticas y glabras en ambas superficies, en tiempo de floración membranosas y verde amarillentas y en la madurez coriáceas y verde pálidas; de pecíolos aplanados o muy ligeramente acanalados. Flores generalmente solitarias, algunas veces en dicasio, la flor central sésil. Frutos globosos a piriformes con sabor dulce a agridulce (Sánchez, 1990).

Características del árbol estudiado

El árbol seleccionado, registro de xiloteca UAMIZ-M12 y herbario UAMIZ-18276 y número de colectores SRD-12 y AQI-5, tuvo una altura total de $9 \mathrm{~m}$.

La corteza externa midió menos de $1 \mathrm{~mm}$ de grosor, estuvo formada por placas que no se desprenden fácilmente, de color castaño (7.5YR 5/6). La corteza interna midió de 4 a $5 \mathrm{~mm}$ de grosor y era de color café rojizo claro (2.5YR 6/4). La albura midió $3.4 \mathrm{~cm}$ en su parte más ancha y en la más angosta $2 \mathrm{~cm}$. El duramen de forma regular, ligeramente excéntrico, midió $15 \mathrm{~cm}$ de diámetro.

Características anatómicas de la madera Macroscópicas (Fig. 2)

La madera presenta diferencia de color entre albura y duramen, el de la albura es castaño claro amarillento (10YR 6/4) y el del duramen presenta tonalidades de color gris oscuro (10YR 4/1) y castaño (10YR 4/3). El olor no es característico y el sabor es amargo. El brillo es mediano, el veteado pronunciado, la textura fina y el hilo recto. Los poros, el parénquima axial y los rayos sólo son visibles con lupa. Las zonas de crecimiento están poco marcadas.

Microscópicas (Figs. 3, 4 y 5)

Los poros presentan distribución difusa, son exclusivamente solitarios, muy numerosos 52 (34-67)/ $/ \mathrm{mm}^{2}$, de diámetro tangencial muy pequeño 37 (22-50) $\mu \mathrm{m}$ y contorno ovalado. Los elementos de vaso son de longitud mediana 688 (350-1200) $\mu \mathrm{m}$, sus paredes presentan puntuaciones areoladas alternas y placa perforada simple. Con gomas. 

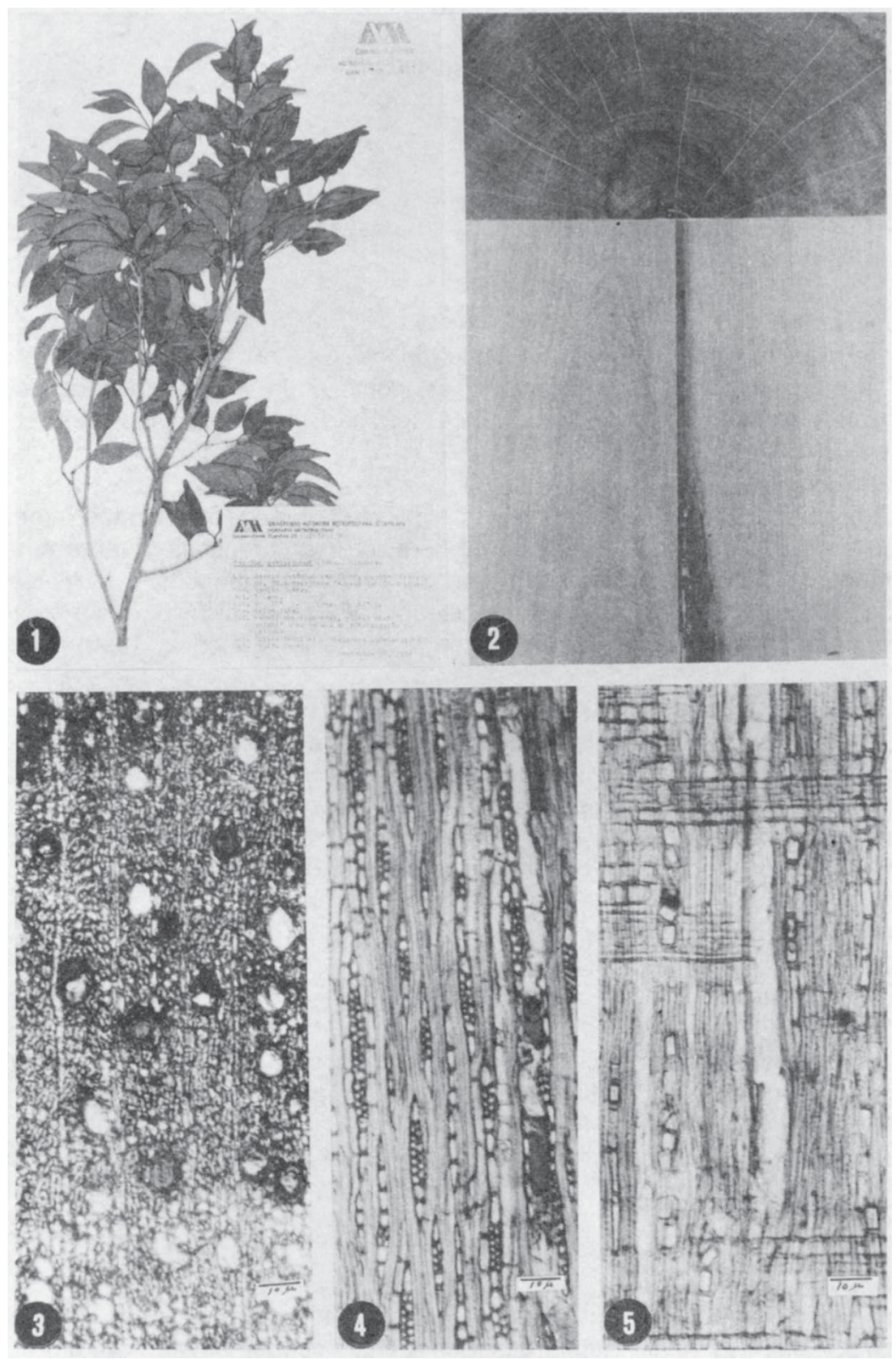

Figs. 1-5. Psidium sartorianum. 1. Muestra de herbario. 2. Tablillas de los tres cortes típicos: tranversal (arriba), tangencial (izquierda) y radial (derecha). 3. Corte transversal. 4. Corte tangencial. 5. Corte radial. 
El parénquima axial es de tipo reticulado con idioblastos que presentan cristales romboidales.

Los rayos son de tipo heterogéneo I y II, muy numerosos $14(11-18) / \mathrm{mm}$, de 2 series (1-2). Los uniseriados son extremadamente bajos $133(60-280) \mu \mathrm{m}$, de 1 a 8 células erectas; los poliseriados son extremadamente bajos 265 (120-480) $\mu \mathrm{m}$, de anchura muy fina 23 (1833) $\mu \mathrm{m}$, los extremos uniseriados de 1 a 8 células. Presentan gomas y cristales romboidales.

Las fibras son de tipo libriforme, de longitud mediana 1208 (750-1760) $\mu \mathrm{m}$, diámetro fino $19(12-25) \mu \mathrm{m}$ y paredes gruesas 7 (3-10) $\mu \mathrm{m}$. Presentan gomas.

Usos

En la zona de recolección la madera se usa para construcción de palapas y en la manufactura de durmientes. En Jalisco para construcción y leña y se sugiere que sea utilizada en muebles y otros artefactos que requieren dureza y buen acabado (Barajas y León, 1989).

\section{Cordia gerascanthus L. Boraginaceae}

Nombres comunes

Baka che, bakal che, bak che, bojom, bojom che, bojon (Sosa et al., 1985).

Distribución en México

Península de Yucatán (Sosa et al., 1985).

Descripción botánica de la especie (Fig. 6)

Arbol de 4 a $30 \mathrm{~m}$ de altura; hojas lanceoladas a elíptico-oblongas, agudas 0 acuminadas y glabras o casi glabras; de pecíolos delgados. Flores blancas, fragantes, sésiles en cimas densas. Frutos elipsoidales con paredes fibrosas encerrando una semilla, permanecen encerrados en el tubo de la corola y el cáliz (Standley, 1924).

Características del árbol estudiado

El árbol seleccionado, registro de xiloteca UAMIZ-M13 y herbario 18275 y número de colectores SRD-13 y AQI-6, tuvo una altura total de $13 \mathrm{~m}$.

La corteza externa midió de 3 a $5 \mathrm{~mm}$ de grosor, estuvo formada por pequeñas placas fácilmente desprendibles, de color castaño rojizo (5YR 4/3 y 5/4). La corteza interna midió de 2 a $5 \mathrm{~mm}$ de grosor y de color gris claro (10YR 7/2). La albura midió $8.3 \mathrm{~cm}$ en su parte más ancha y en la más angosta $1.7 \mathrm{~cm}$. El duramen, de forma muy irregular y totalmente excéntrico, midió en su parte más ancha $15 \mathrm{~cm}$ y en la más angosta $13 \mathrm{~cm}$ de diámetro.

Características anatómicas de la madera

Macroscópicas (Fig. 7)

La madera presenta diferencia de color entre albura y duramen, el de la primera es castaño muy pálido (10YR 7/4) y el del segundo presenta tonalidades de color gris muy oscuro (10YR 5/3) y castaño muy pálido (10YR 6/3). El olor y el sabor no son característicos. El brillo es mediano, el veteado pronunciado, la textura mediana y el hilo recto. Los poros, 
el parénquima axial y las fibras son fácilmente visibles con lupa. Las zonas de crecimiento están marcadas por bandas de fibras y poros.

Microscópicas (Figs. 8, 9 y 10)

Los poros presentan distribución difusa, en su mayoría son solitarios, algunos múltiples radiales de 2-4, agrupados en 3 y raramente en 6 , moderadamente numerosos $17(10-25) / \mathrm{mm}^{2}$, de diámetro tangencial moderadamente pequeño 79 (25-125) um y contorno ovalado. Los elementos de vaso son de longitud moderadamente corta 252 (90350) $\mu \mathrm{m}$, sus paredes presentan puntuaciones areoladas alternas y placa perforada simple. Con gomas y tílides.

El parénquima axial es de tipo vasicéntrico, difuso y marginal.

Los rayos son de tipo heterogéneo II y III, moderadamente numerosos $6(4-8) / \mathrm{mm}$, de 4 series (2-5), son muy bajos 761 (230-1900) $\mu \mathrm{m}$, de anchura mediana 77 (25-163) $\mu \mathrm{m}$, los extremos de 1 a 2 células erectas y una capa de células envolventes. Presentan cristales romboidales, principalmente en las células erectas.

Las fibras son de tipo libriforme, de longitud mediana $1460(900-2100) \mu \mathrm{m}$, de diámetro fino $15(10-23) \mu \mathrm{m}$ y paredes gruesas 5 (3-8) $\mu \mathrm{m}$.

Usos

En la zona de recolección la madera se usa para la fabricación de muebles, especialmente para sillas.

\section{DISCUSION}

Las maderas estudiadas de Psidium sartorianum y Cordia gerascanthus son semejantes en las siguientes características macroscópicas: el color, que en ambas se presenta como tonalidades oscuras en el duramen y claro en la albura, por lo que sus veteados son pronunciados; los tamaños de sus elementos constitutivos que influyen en la textura fina así como hilo recto, brillo mediano y olor no perceptible.

En cuanto a las características microscópicas, ambas especies presentan similitud en su porosidad difusa con poros muy numerosos, solitarios y de diámetro pequeño, sus elementos de vaso tienen puntuaciones areoladas alternas y placa perforada simple; las fibras son de tipo libriforme, medianas, de paredes gruesas y de diámetro fino.

La madera de Psidium sartorianum procedente de Quintana Roo difiere de la de $P$. sartorianum de Jalisco, estudiada por Barajas y León (1989), en las siguientes características macroscópicas y microscópicas. La de Quintana Roo presenta sabor amargo, textura fina e hilo recto, porosidad difusa, parénquima axial reticulado, rayos heterogéneos tipos I y II y fibras de longitud mediana, en contraste con la de Jalisco que no tiene sabor característico, la textura es muy fina, el hilo es ondulado a ligeramente entrecruzado, la porosidad semianular, el parénquima axial apotraqueal en bandas y paratraqueal escaso, los rayos heterogéneos tipos II y III y las fibras moderadamente largas. Estas diferencias podrían estar relacionadas con el tipo de vegetación, pues los árboles de las especies de este estudio crecen en una selva mediana subperennifolia y los de Jalisco en una selva baja caducifolia. 
Rebollar et al.: Anatomía de la Madera de Psidium sartorianum y Cordia gerascanthus



Fig. 6-10. Cordia gerascanthus. 6. Muestra de herbario. 7. Tablillas de los tres cortes típicos: transversal (arriba), tangencial (izquierda) y radial (derecha). 8. Corte transversal. 9. Corte tangencial. 10. Corte radial. 


\section{AGRADECIMIENTOS}

Se agradece al Centro de Investigaciones de Quintana Roo, en especial al personal del Jardín Botánico, por las facilidades brindadas en el trabajo de colecta. Al M.en C. Adolfo Espejo Serna por la identificación del material de herbario; al Sr. Jorge Lodiggiani por el trabajo fotográfico y al M. en C. José Luis Córdova Frunz por el diseño del programa estadístico, estos tres últimos de la Universidad Autónoma Metropolitana-Iztapalapa.

\section{LITERATURA CITADA}

Andrade, D. P. 1976. Aspectos ecológicos y usos de veintidos especies maderables de la zona lacandona de Chiapas. Tesis Profesional. Facultad de Ciencias. Universidad Nacional Autónoma de México. México, D.F. 158 pp.

Barajas, J. 1981. Descriptions and notes on the wood anatomy of Boraginaceae from Western Mexico. IAWA Bull. n.s. 2(2-3): 61-67.

Barajas, J. y R. Echenique. 1976. Anatomía de maderas de México. I. Doce especies de Jalisco y Veracruz. México. Biótica 1(2): 29-57.

Barajas, J. y C. León. 1989. Anatomía de maderas de México: especies de una selva baja caducifolia. Publicaciones Especiales I. Instituto de Biología. Universidad Nacional Autónoma de México. México, D.F. 128 pp.

Chattaway, M. 1932. Proposed standards for numerical values used in describing woods. Trop. Woods 29: $20-28$.

Escalante, S. 1986. La flora del Jardín Botánico del Centro de Investigaciones de Quintana Roo A. C. Tesis Profesional. Facultad de Ciencias Biológicas. Universidad Veracruzana. Xalapa, Veracruz. $163 \mathrm{pp}$.

Guridi, L. 1968. Anatomía de la madera de cinco especies tropicales de importancia económica. Tesis Profesional. Facultad de Ciencias. Universidad Nacional Autónoma de México. México, D.F. $34 \mathrm{pp}$.

Guridi, L. 1980. La madera de las artesanías del estado de Michoacán. Bol. Div. Inst. Nac. Invest. For. México No. 50.120 pp.

IAWA Committee. 1937. Standard terms of length of vessel members and wood fibers. Trop. Woods 51: 21.

IAWA Committee. 1939. Standard terms of size for vessel diameter and ray width. Trop. Woods 59: $51-52$

IAWA Committee. 1989. IAWA list of microscopic features for hardwood identification. IAWA Bull. n.s. 10(3): 219-332.

Johansen, A. 1940. Plant microtechnique. McGraw-Hill. New York. 523 pp.

Kribs, D. 1968. Commercial foreign woods on the American market. Dover Publications Inc. New York. $241 \mathrm{pp}$.

Kukachka, B. 1977. Sectioning refractory woods for anatomical studies. Forest Service Research Note FPL-0236: $1-9$.

León, C. y J. Barajas. 1987. Variación de la estructura de la madera en Cordia elaeagnoides DC. Biótica 12(2): 121-129.

Miranda, F. 1978. Vegetación de la Península Yucateca. 2a. Impresión, Colegio de Postgraduados, SARH. Chapingo. México. pp. 161-271.

Munsell Color Company. 1954. Munsell soil color charts. Baltimore, Maryland. 17 pp.

Ortega, F. 1984. Anatomía de la madera de seis especies de la selva alta perennifolia. Tesis Profesional. Facultad de Ciencias. Universidad Nacional Autónoma de México. México, D.F. 68 pp. 
Ortega, F., L. Guerrero, T. Carmona y C. Córdova. 1988. Angiospermas arbóreas de México. Núm. 1. Anatomía de la madera de 28 especies de Cosautlán de Carvajal, Veracruz. Boletín Técnico La Madera y su Uso No. 19. Instituto Nacional de Investigaciones sobre Recursos BióticosUniversidad Autónoma Metropolitana Atzcapotzalco. México. D.F. 206 pp.

Ortega, M. 1958. Estructura anatómica e histológica de un grupo de 28 especies del bosque chiapaneco. Tesis Profesional. Facultad de Ciencias. Universidad Nacional Autónoma de México. México, D.F. $241 \mathrm{pp}$.

Pennington, T. D. y J. Sarukhán. 1968. Arboles tropicales de México. Instituto Nacional de Investigaciones Forestales y Organización de las Naciones Unidas para la Agricultura y la Alimentación. México, D.F. 413 pp.

Ramos, C. y V. Díaz. 1981. Instrucciones para colectar muestras de madera para estudios tecnológicos. Boletín Divulgativo Instituto Nacional de Investigaciones Forestales No. 54. México, D.F. 15 pp.

Rebollar, S., C. de la Paz Pérez y A. Quintanar. 1987. Maderas de la Península de Yucatán, México. 1. Estudio anatómico de la madera de tres especies del estado de Yucatán. Biótica 12(3): 159179.

Sánchez, P. E. 1990. Myrtaceae. Flora de Veracruz. Fascículo 62.146 pp.

Sosa, V., J. S. Flores, V. Rico-Gray, R. Lira y J. J. Ortiz. 1985. Etnoflora Yucatanense, Fascículo 1. Lista florística y sinonimia maya. Instituto Nacional de Investigaciones sobre Recursos Bióticos. Xalapa, Veracruz. 225 pp.

Standley, P. C. 1924. Trees and shrubs of Mexico. Boraginaceae. Contr. U. S. Nat. Herb. 23(4): 12161234.

Tortorelli, L. 1956. Maderas y bosques argentinos. Acme. Buenos Aires. 910 pp. 